\title{
Design of Extended Depth-of-Focus Laser Beams Using Orthogonal Beam Expansions
}

\author{
David P. Goren \\ Symbol Technologies Inc., One Symbol Plaza, Holtsville, NY 11742-1300, USA \\ Email: gorens@optonline.net \\ Joseph Katz \\ Symbol Technologies Inc., One Symbol Plaza, Holtsville, NY 11742-1300, USA \\ Mitsubishi Electric Research Laboratories, 201 Broadway, Cambridge, MA 02139, USA \\ Email:katz@merl.com
}

Leonard Bergstein

Symbol Technologies Inc., One Symbol Plaza, Holtsville, NY 11742-1300, USA

Email:drssb2000@aol.com

Received 16 April 2004; Revised 3 November 2004

\begin{abstract}
Laser beams with extended depth of focus have many practical applications, such as scanning printed bar codes. Previous work has concentrated on synthesizing such beams by approximating the nondiffracting Bessel beam solution to the wave equation. In this paper, we introduce an alternate novel synthesis method that is based on maintaining a minimum MTF value (contrast) over the largest possible distance. To achieve this, the coefficients of an orthogonal beam expansion are sequentially optimized to this criterion. One of the main advantages of this method is that it can be easily generalized to noncircularly symmetrical beams by the appropriate choice of the beam expansion basis functions. This approach is found to be very useful for applications that involve scanning of the laser beam.
\end{abstract}

Keywords and phrases: lasers, beam shaping, optical transfer functions, propagation, extended depth of focus, orthogonal beam expansion.

\section{INTRODUCTION}

Laser beams are commonly used to read digital information that has been encoded as a sequence of alternating light and dark regions on a reflective media. One such application is the reading of printed bar codes [1]. In this application, the distance from the bar code reader to the bar code label is usually variable and unknown, and in many cases it is desirable to read it over the largest possible distance, a feature that greatly improves the ergonomics of the reader and reduces operator training.

For such a system, the laser beam should have a large depth of focus, loosely defined as the region where the beam is "narrow enough" to resolve the fine structure of the bar code. A proper definition of the depth of focus is crucial to optimize such a system, and it must take into account that the spatially encoded information can typically withstand moderate distortions and still be properly decoded. In addition, when scanning bar codes whose aspect ratio (i.e., the ratio between the height and width of a bar code element) is high, it is the line-spread function (LSF) of the beam, rather than its point-spread function (PSF), that determines the overall system performance. In such cases, a highly elliptical beam is desirable in order to take advantage of the vertical redundancy in the code, and to reduce printing or laser speckle noise. (In the case of two-dimensional bar codes [1], a nearly circular beam is required in order to avoid interrow interference.)

Most previous work on synthesizing extended depth-offocus laser beams have been based on either approximating the nondiffracting Bessel beam solution to the wave equation $[2,3,4]$, or applying more general $3 \mathrm{D}$ synthesis techniques $[5,6]$. Compared to the more general 3D synthesis techniques, the technique developed in this paper uses a simpler optimization criterion, and does not require samples of the desired beam profile at various planes. In addition, the use of a modal beam expansion guarantees the resulting beam satisfies the paraxial wave equation.

The outline of this paper is as follows. First an appropriate definition of depth of focus based on the modulation 


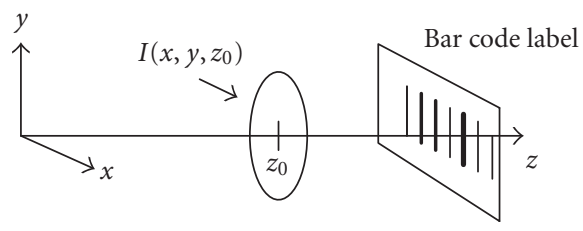

FIGURE 1: Schematic layout of the optical system.

transfer function (MTF) is presented. Using this definition, a Gaussian beam is optimized to achieve a maximum depth of focus, followed by the optimization of more general beams through the use of orthogonal beam expansions. Both circular and noncircular beams are considered by using the appropriate basis functions.

\section{MTF-BASED OPTIMIZATION CRITERION}

Consider a two-dimensional laser beam intensity profile at a fixed distance $z_{0}$ given by $I\left(x, y, z_{0}\right)$ with Fourier transform $U\left(u, v, z_{0}\right)$, where $v$ is spatial frequency and $x, y$, and $z$ are distances in the $x$-, $y$-, and $z$-directions, respectively, of the beam profile. The overall setup of the system is shown schematically in Figure 1. The line-spread function (LSF) of the beam is defined as [7]

$$
s\left(x, z_{0}\right)=\int_{-\infty}^{\infty} I\left(x, y, z_{0}\right) d y
$$

The modulation transfer function (MTF) of the beam is defined as the Fourier transform of the LSF and can be expressed as

$$
F\left(u, z_{0}\right)=U\left(u, 0, z_{0}\right)=\int_{-\infty}^{\infty} s\left(x, z_{0}\right) e^{-j u x} d x
$$

The MTF describes the spatial filtering effect of scanning the laser beam over a one-dimensional spatial pattern extended infinitely in the $y$-direction.

An important class of (approximately) such spatial patterns is the ubiquitous printed bar code. These signals encode digital information through the use of alternating white and dark regions (bars and spaces) of varying widths. Laser scanning systems designed to read such patterns typically require a minimum contrast level for all spatial frequencies up to the highest fundamental spatial frequency of the narrowest bar/space pair. Higher signal-to-noise ratios allow lower contrast levels to be used.

For such applications, it is convenient to define the depth of focus of a scanning laser beam to be the region on the $z$ axis that maintains a minimum contrast level, $C$, for all frequencies up to and including the highest fundamental frequency. In what follows, all MTF curves will be normalized so that $F(u=0, z)=1$ for all $z$.

Let $u_{0}$ denote the fundamental spatial frequency of the narrowest elements of a bar code (i.e., narrow bar/space

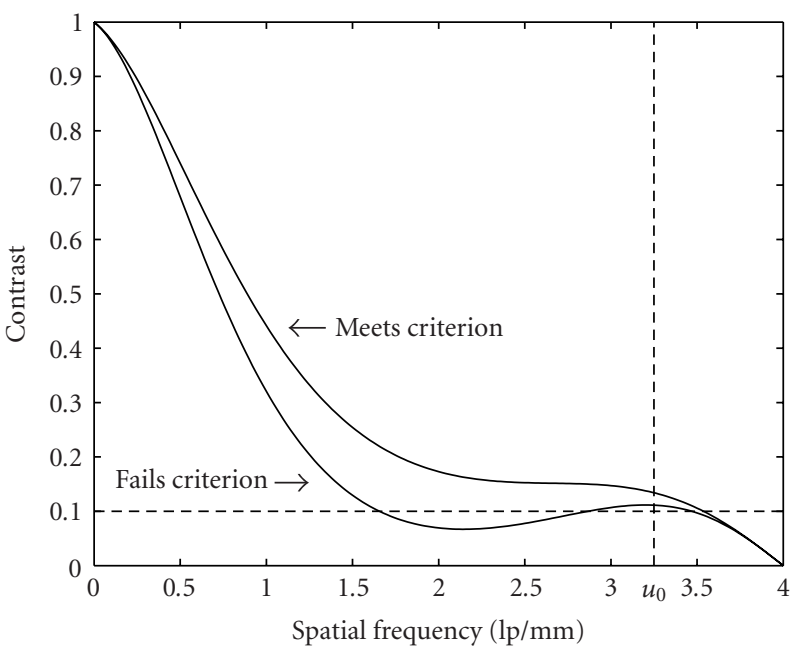

FIgURE 2: Example of MTF curves.

pair). Such a spatial pattern will be considered within focus at a distance $z_{0}$ if

$$
F\left(u, z_{0}\right) \geq C, \quad 0 \leq u \leq u_{0} .
$$

This criterion guarantees that there will be an adequate level of signal modulation present at the highest fundamental bar/space frequency $u_{0}$ and that no contrast reversals or loss of modulation occurs at lower frequencies. An illustration of MTF curves that both meet and fail the criterion is shown in Figure 2.

This definition of depth of field will be used to design laser beams that maximize the depth of focus given a highest spatial frequency $u_{0}$ and the minimum contrast level $C$.

\section{GAUSSIAN BEAM OPTIMIZATION}

The optimization of a simple Gaussian beam [8] will serve both as a simple demonstration of the method, as well as the first step required for the optimization of more general beams. Note that the LSF of a two-dimensional Gaussian beam is also Gaussian, thus a one-dimensional beam optimization is sufficient.

The LSF of a Gaussian beam at a fixed distance $z_{0}$ is given by

$$
s\left(x, z_{0}\right)=\frac{\sqrt{2}}{\sqrt{\pi} \omega\left(z_{0}\right)} e^{-2 x^{2} / \omega^{2}\left(z_{0}\right)},
$$

where $\omega\left(z_{0}\right)$ is the beam radius at $z_{0}$ given by

$$
\omega\left(z_{0}\right)=\omega_{0} \sqrt{1+\left(\frac{\lambda z_{0}}{\pi \omega_{0}^{2}}\right)^{2}} .
$$

For this case, it is assumed that the only free parameter to optimize is the minimum beam radius $\omega_{0}$ occurring at $z=0$. 
The optimum beam radius $\omega_{0}$ that maximizes the depth of focus for a given contrast $C$ and maximum spatial frequency $u_{0}$ is calculated to be

$$
\omega_{0}=\frac{1}{u_{0} \pi} \sqrt{-\ln C}
$$

The resulting depth of focus is

$$
d_{\mathrm{opt}}=\frac{-2 \ln C}{u_{0}^{2} \pi \lambda}
$$

with the distances $|z| \leq d_{\mathrm{opt}} / 2$ meeting the criterion for focus as defined by (3). Note that the depth of focus is inversely proportional to the square of the maximum spatial frequency $u_{0}$. This inverse relationship also applies to higherorder modes.

In the following section, the optimization criterion will be applied to the more general set of Hermite-Gaussian beams.

\section{OPTIMIZATION OF THE HERMITE-GAUSSIAN EXPANSION}

The Gaussian beam is the lowest-order member of the family of Hermite-Gaussian (H-G) beams (also known as H-G modes [8]). The H-G beams form a complete orthogonal set of functions satisfying the paraxial wave equation in rectangular coordinates, and are thereby capable of representing an arbitrary propagating beam. These beams also have the desirable property that they remain $\mathrm{H}-\mathrm{G}$ as they propagate, thus providing a simple model for the propagation and optimization of complex beams. A description of the H-G beams is given in Appendix A.

Because the described optimization criterion is solely a function of the LSF, and the fact that the two-dimensional $\mathrm{H}$ $\mathrm{G}$ functions are separable in rectangular coordinates, we only need to consider a one-dimensional expansion. We will restrict the generated beam to certain desirable symmetries appropriate to real-world applications. The first is that the LSF of the beams are symmetrical about $x=0$. This condition is guaranteed by using only even-order $\mathrm{H}-\mathrm{G}$ modes in the beam expansion. The second symmetry is that the beam propagates symmetrically about $z=0$. This restricts the expansion coefficients to be real, and guarantees maximum depth of field for a chosen $u_{0}$.

The optimizing approach is based on representing the desired beam as a sum of H-G beams with unknown coefficients. In general, an infinite sum is required to represent an arbitrary beam. We will show that in practical applications, the series needs to include only a relative small number of $N$ terms. This leaves $N$ unknown parameters to be optimized. A finite $\mathrm{H}-\mathrm{G}$ expansion of a one-dimensional beam (within a constant factor) using $N$ even-order modes is given by

$$
g_{N}(x, z)=u_{0}\left(x, z ; b_{0}\right)+\sum_{n=1}^{N-1} A_{n} u_{2 n}\left(x, z ; b_{0}\right)
$$

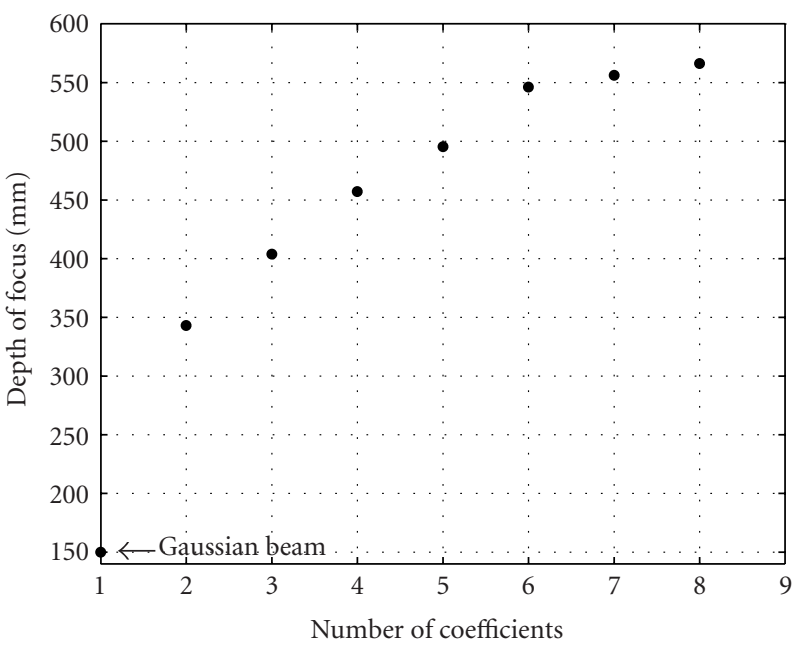

Figure 3: Depth of focus versus number of H-G coefficients.

where the $N$ unknown parameters to be optimized are $\left[b_{0}, A_{1}, A_{2}, \ldots, A_{N-1}\right]$. The parameter $b_{0}$ is the confocal parameter and is shared by all the modes (see Appendix A). It should be noted that for Gaussian beams that correspond to the lowest order of the $\mathrm{H}-\mathrm{G}$ family of beams, this parameter is known as the "Rayleigh distance." The LSF of the beam at a distance $z_{0}$ is then given by the beam intensity expressed as

$$
s\left(x, z_{0}\right)=g_{N}\left(x, z_{0}\right) \cdot g_{N}^{*}\left(x, z_{0}\right) .
$$

As more terms of the series are included in the optimization, the depth of focus of the beam increases.

Optimization of the beam parameters was performed with computer search techniques using a sequential series of optimizations for increasing values of $N$ (see Matlab Optimization Toolbox at http://www.mathworks.com/ products/optimization). In particular, the multidimensional simplex search algorithm of Nelder and Mead was used due to the difficulty of obtaining accurate derivative information for the calculated depth of field.

The optimization for $N=1$ (Gaussian beam) was analytically derived in Section 3. For $N>1$, the critical initial guess required to seed the numerical multidimensional optimization of all $N$ parameters was supplied by the previous $(N-1)$ term optimization. In addition, it was found useful to refine the initial guess by first performing a suboptimization using only the newly added coefficient together with the confocal parameter. This procedure greatly enhanced the convergence to the appropriate solution. It is important to note that all frequencies from 0 to $u_{0}$ must be checked at every $z$ to determine if the beam is within focus as defined in (3).

The depth of focus achievable as a function of the number of terms in the series is shown in Figure 3 for a maximum spatial frequency of $3.9 \mathrm{lp} / \mathrm{mm}$ (e.g., bar code with a 5mil narrow element) and a minimum contrast of $10 \%$. The numerical values of the optimized parameters are given in Table 1. 
TABle 1: H-G coefficients.

\begin{tabular}{ccccccccc}
\hline Number of terms $N$ & $b_{0}$ & $A_{1}$ & $A_{2}$ & $A_{3}$ & $A_{4}$ & $A_{5}$ & $A_{6}$ & $A_{7}$ \\
\hline 1 & 0.07 & - & - & - & - & - & - \\
2 & 0.24 & -1.19 & - & - & - & - & - \\
3 & 0.33 & -1.08 & 0.55 & - & - & - & - \\
4 & 0.53 & -0.9 & 0.78 & -0.49 & - & - & - \\
5 & 0.85 & -0.87 & 0.78 & -0.57 & 0.70 & - & - \\
6 & 1.08 & -1.03 & 0.91 & -0.83 & 0.77 & -0.7 & - \\
7 & 1.36 & -1.0 & 1.0 & -0.88 & 0.74 & -0.73 & 0.91 & - \\
8 & 1.60 & -0.96 & 1.0 & -0.96 & 0.78 & -0.67 & 0.80 & -0.75 \\
\hline
\end{tabular}

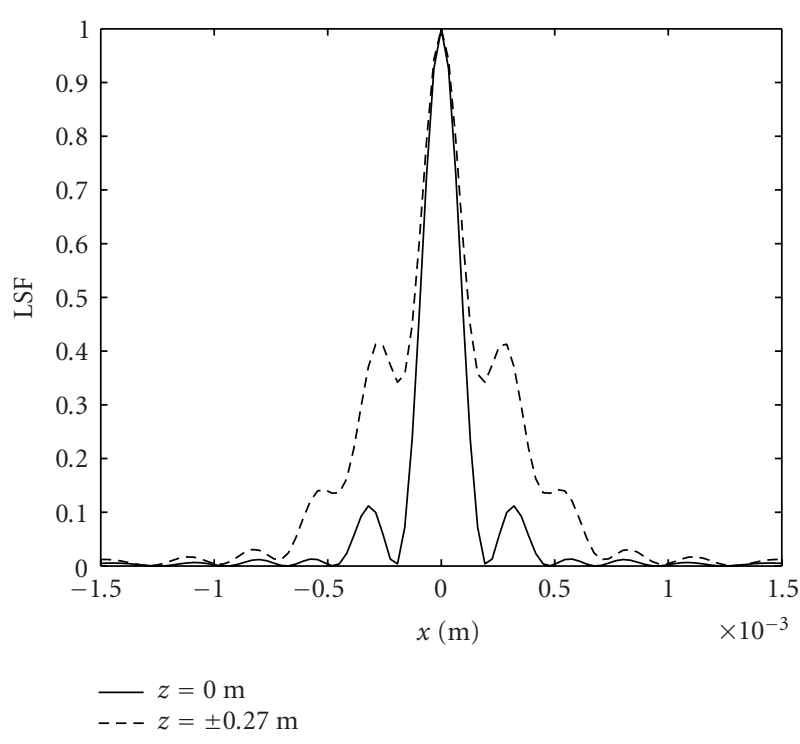

Figure 4: LSF curves (H-G).

Examining Figure 3 shows that simply adding a second term to the series (Gaussian beam and the next lowest evenorder mode) doubles the depth of focus. As more terms are added, the rate of improvement decreases. In particular, there is little benefit after six terms. Figures 4 and 5 show the resulting LSF and MTF, respectively, of a beam that uses six terms. To physically realize the beam, diffractive optical elements can be employed [9], with higher-order beams requiring more spatial resolution.

\section{OPTIMIZATION OF THE LAGUERRE-GAUSSIAN EXPANSION}

In the previous section, we used the essentially onedimensional $\mathrm{H}-\mathrm{G}$ expansion to optimize optical beams used to read conventional bar codes. In the case of a twodimensional bar code, a different optimization criterion is needed, since elongated optical beams cause severe interrow interference. In this case, it is advantageous to use optical beams with circular symmetry. In this section, we will analyze one example based on the Laguerre-Gaussian series [10].

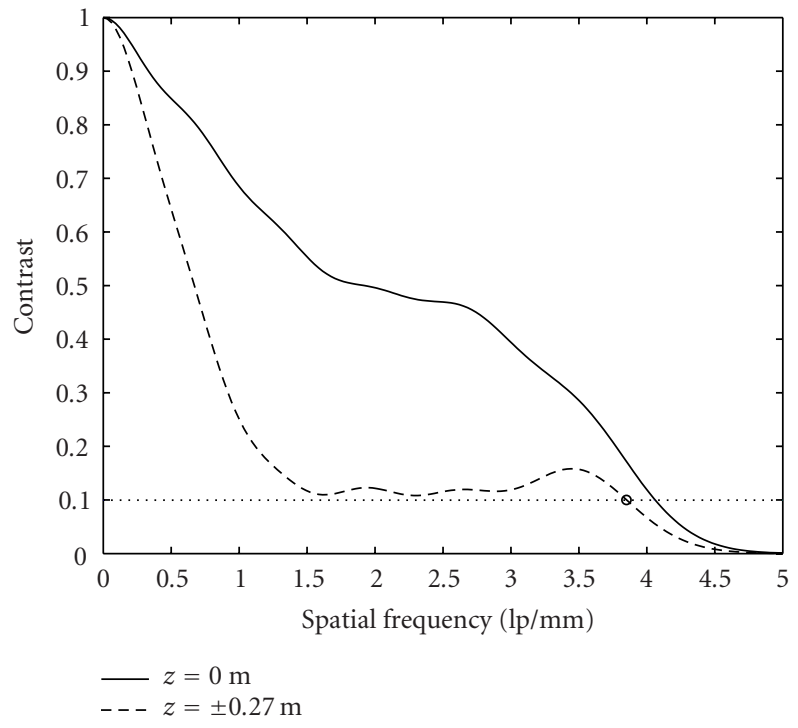

Figure 5: MTF curves (H-G).

In spherical coordinates, the family of beams that form a complete set of solutions to the paraxial wave equation are the Laguerre-Gaussian (L-G) beams. A description of the L-G beams are given in Appendix B. In this case, the LSF can be expressed as

$$
s\left(x, z_{0}\right)=\int_{-\infty}^{\infty} I\left(\sqrt{x^{2}+y^{2}}, z_{0}\right) d y .
$$

In a fashion similar to the optimization of the H-G beams, the coefficients of a six-term L-G expansion were optimized to guarantee a contrast level of $10 \%$ at $3.94 \mathrm{lp} / \mathrm{mm}$. The resulting LSF and MTF curves are shown in Figures 6 and 7, respectively. The optimized parameters are given in Table 2.

It should be noted that while both the circularly symmetric optimization (L-G) and the 1D (H-G) optimization maintain a minimum contrast level, the circular beam's LSF undergoes significantly less variation over the depth of focus compared to the $1 \mathrm{D}$ beam, as evidenced in the curves presented in Figure 4 compared to those of Figure 6 . This is due to the existence of a nondiffracting beam solution in spherical coordinates while no such beam exists in rectangular coordinates [2]. 
TABLE 2: L-G coefficients.

\begin{tabular}{ccccccc}
\hline Number of terms $N$ & $b_{0}$ & $A_{1}$ & $A_{2}$ & $A_{3}$ & $A_{4}$ & $A_{5}$ \\
\hline 1 & 0.07 & - & - & - & - & - \\
2 & 0.21 & 0.70 & - & - & - & - \\
3 & 0.64 & 1.58 & 1.29 & - & - & - \\
4 & 0.82 & 1.35 & 1.66 & 1.46 & - & - \\
5 & 0.98 & 1.25 & 1.60 & 1.87 & 1.17 & 1.17 \\
6 & 1.11 & 1.17 & 2.07 & 3.35 & 2.03 & - \\
\hline
\end{tabular}

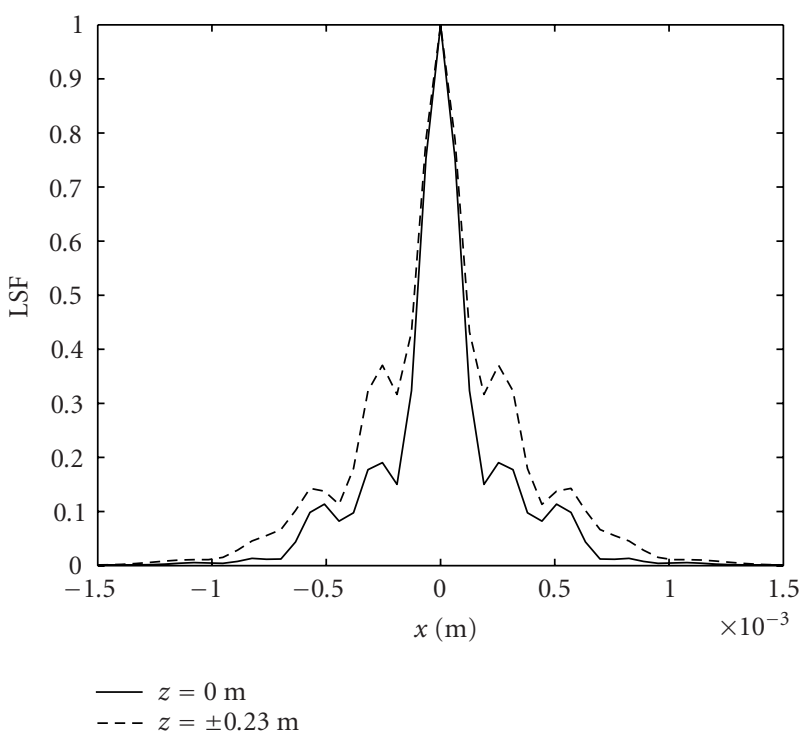

Figure 6: LSF curves (L-G).

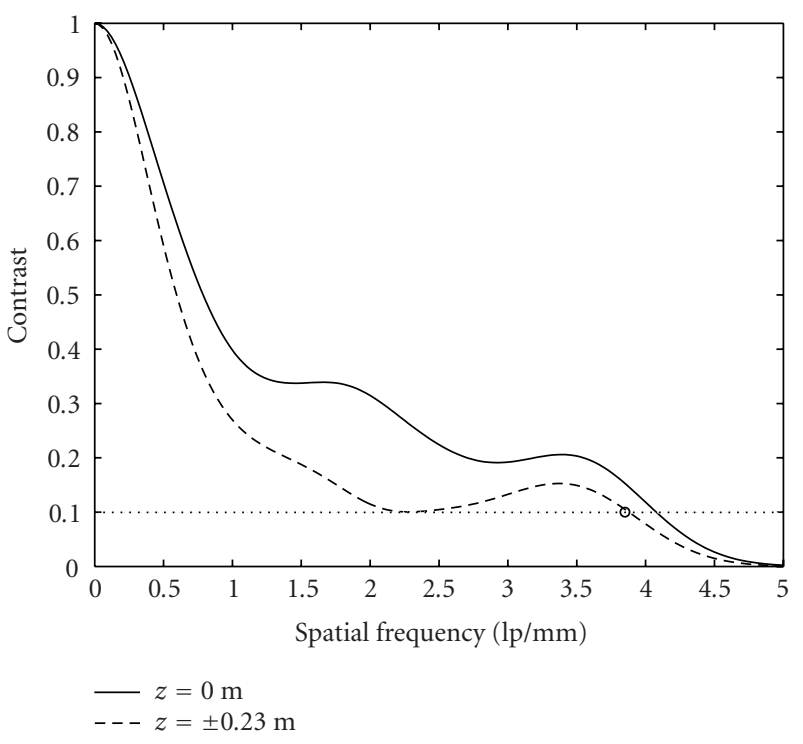

Figure 7: MTF curves (L-G).

\section{SUMMARY AND CONCLUSIONS}

Extended depth-of-focus laser beams have many practical applications. One such application is the scanning and decoding of printed bar codes over extended ranges. Previous work has concentrated on synthesizing such beams by approximating the nondiffracting Bessel beam solution to the wave equation $[3,4]$. In this paper, a novel optimization technique was presented based on orthogonal beam expansions. One of the main advantages of this method is that it can generate noncircularly symmetrical beams which offer great advantage when scanning noisy one-dimensional patterns such as bar codes. Using this expansion, we have shown that with a relatively small number of terms, the laser beam operational depth of focus can be readily extended by more than threefold.

It is important to note that the overall performance of a bar reader depends not only on the optical properties of its optical beam, but also on the processing of the signal derived from the reflected beam.

When applying the beams described in this paper to bar code scanning applications, the price to be paid for the extended depth of focus is lower contrast (less optical power received) and waveform complexity resulting from the sidelobe structure of the beam. Higher-power lasers along with lower- noise electronics can address the contrast issue and more advanced signal processing techniques are required to remove the resulting distortion artifacts and to correctly reconstruct the original spatial pattern $[11,12]$.

\section{APPENDICES}

\section{A. HERMITE-GAUSSIAN MODES}

A Hermite-Gaussian function of order $n$ is expressed as

$$
\psi_{n}(\xi)=H_{n}(\xi) e^{-\xi^{2} / 2}
$$

where $H_{n}(\xi)$ are Hermite polynomials. Some examples of low-order Hermite polynomials are

$$
H_{0}(\xi)=1, \quad H_{1}(\xi)=2 \xi, \quad H_{2}(\xi)=4 \xi^{2}-2 .
$$

The $n$ th-order Hermite-Gaussian mode is defined as

$$
u_{n}\left(x, z=0, b_{0}\right)=C_{n} \psi_{n}\left(\frac{\sqrt{2} x}{\omega_{0}}\right)
$$


with

$$
\omega_{0}=\sqrt{\frac{2 b_{0}}{k}}
$$

where $b_{0}$ is the confocal parameter and $k$ is the wave number. For Gaussian beams, the lowest order of the H-G beams, the confocal parameter $b_{0}$ is also known as the Rayleigh distance so that $\omega\left(b_{0}\right)=\sqrt{2} \omega_{0}$.

$C_{n}$ is the energy normalization constant given by

$$
C_{n}=\left(\frac{\sqrt{2}}{\omega_{0} 2^{n} n ! \sqrt{\pi}}\right)^{1 / 2}
$$

For $z \neq 0$, the propagation of the Hermite-Gaussian modes are given by

$$
\begin{aligned}
u_{n}\left(x, z ; b_{0}\right)= & e^{j(k z-\pi / 4)} \frac{C_{n}}{\left(1+z^{2} / b_{0}^{2}\right)^{1 / 4}} \psi_{n}\left(\frac{\sqrt{2} x}{\omega(z)}\right) \\
& \times e^{-j k x^{2} / 2 R(z)} e^{j(2 n+1 / 2) \phi(z)},
\end{aligned}
$$

where

$$
\begin{array}{r}
\omega(z)=\sqrt{\frac{2 b_{0}}{k}\left(1+\frac{z^{2}}{b_{0}^{2}}\right),} \\
R(z)=\frac{z^{2}+b_{0}^{2}}{z}, \\
\phi(z)=\tan ^{-1}\left(\frac{z}{b_{0}}\right) .
\end{array}
$$

Consider an arbitrary one-dimensional beam with input amplitude function $h(x)$ at $z=0$. The function $h(x)$ can be expanded as

$$
h(x)=\sum_{n=0}^{\infty} A_{n} u_{n}\left(x, z=0 ; b_{0}\right)
$$

where $A_{n}$ are the expansion coefficients and $b_{0}$ is the confocal parameter shared by all the H-G modes. The propagation of the beam for $z \neq 0$ can then be expressed as a summation of the individual propagating beams given by

$$
g(x, z)=\sum_{n=0}^{\infty} A_{n} u_{n}\left(x, z ; b_{0}\right)
$$

The intensity profile at a distance $z_{0}$ is given by

$$
I\left(x, z_{0}\right)=g\left(x, z_{0}\right) g^{*}\left(x, z_{0}\right)
$$

\section{B. LAGUERRE-GAUSSIAN MODES}

The $n$ th-order Laguerre-Gaussian mode at $z=0$ is defined as

$$
u_{k}\left(r, z=0 ; b_{0}\right)=\frac{1}{\sqrt{\pi}} L_{k}\left(\frac{2 r^{2}}{\omega_{0}^{2}}\right) e^{-r^{2} / \omega_{0}^{2}},
$$

where $\omega_{0}$ is define in (A.4) and $l_{k}(\xi)$ are Laguerre polynomials. Some examples of low-order Laguerre polynomials are

$$
l_{0}(\xi)=1, \quad l_{1}(\xi)=-\xi+1, \quad l_{2}(\xi)=\xi^{2}-4 \xi+2 .
$$

For $z \neq 0$, the propagation of the Laguerre-Gaussian modes is given by

$$
\begin{aligned}
u_{k}\left(x, z ; b_{0}\right)= & e^{j(k z)} \frac{1}{\sqrt{\pi}} \frac{1}{\left(1+z^{2} / b_{0}^{2}\right)^{1 / 2}} L_{k}\left(\frac{2 r^{2}}{\omega^{2}(z)}\right) \\
& \times e^{-r^{2} / \omega_{0}^{2}} e^{-j k x^{2} / 2 R(z)} e^{j(2 k+1) \phi(z)},
\end{aligned}
$$

where $\omega(z), R(z)$, and $\phi(z)$ are defined in (A.7).

\section{DISCLAIMER}

This paper reflects the personal understandings and opinions of the authors only and is not intended in any way to convey any position, policy, or opinion of the author's employer, Symbol Technologies, Inc. The authors and Symbol Technologies, Inc. disclaim any liability for any errors or omissions in the paper.

\section{ACKNOWLEDGMENT}

The authors would like to acknowledge Emanuel Marom, Ed Barkan, and Vladimir Gurevich for useful technical discussions.

\section{REFERENCES}

[1] R. C. Palmer, The Bar Code Book, Helmers Publishing, Petersborough, NH, USA, 1995.

[2] J. Durnin, "Exact solutions for nondiffracting beams. I. The scalar theory," Journal of the Optical Society of America $\{A\}$, vol. 4, no. 4, pp. 651-654, 1987.

[3] Y. Li, V. Gurevich, M. Krichever, J. Katz, and E. Marom, "Propagation of anisotropic Bessel-Gaussian beams: sidelobe control, mode selection, and field depth," Applied Optics, vol. 40, no. 16, pp. 2709-2721, 2001.

[4] K. Tanaka, M. Tguchi, and T. Tanaka, "Quasi-diffraction-free beams," Journal of the Optical Society of America $\{A\}$, vol. 18, no. 7, pp. 1644-1649, 2001.

[5] R. Piestun and J. Shamir, "Synthesis of three-dimensional light fields and applications," Proc. IEEE, vol. 90, no. 2, pp. 222-244, 2002.

[6] U. Levy, D. Mendlovic, Z. Zalevsky, G. Shabtay, and E. Marom, "Iterative algorithm for determining optimal beam profiles in a three-dimensional space," Applied Optics, vol. 38, no. 32, pp. 6732-6736, 1999.

[7] A. Papoulis, Systems and Transforms with Applications in Optics, McGraw Hill Book Company, New York, NY, USA, 1968. 
[8] H. A. Haus, Waves and Fields in Optoelectronics, Prentice Hall, Englewood Cliffs, NJ, USA, 1984.

[9] E. Marom, D. Mendlovic, N. Konforti, J. Katz, and C. Tan, "Diffractive optic elements for forming scanning beams", in Diffractive and Holographic Device Technologies and Applications IV, vol. 3010 of Proceedings of SPIE, San Jose, Calif, USA, February 1997.

[10] A. E. Siegman, Lasers, University Science Books, Mill Valley, Calif, USA, 1986.

[11] S. J. Shellhammer, D. P. Goren, and T. Pavlidis, "Novel signalprocessing techniques in barcode scanning," IEEE Robot. Automat. Mag., vol. 6, no. 1, pp. 57-65, 1999.

[12] S. J. Shellhammer and D. Goren, "Fuzzy logic bar code scanners," in Proc. IEEE Workshop on Automatic Identification Advanced Technologies (WAIAT '97), pp. 49-52, Stony Brook, NY, USA, November 1997.

David P. Goren received his B.S. degree in electrical engineering from SUNY at Stony Brook in 1986. He received his M.S. degree in electrical engineering in 1991 from Polytechnic University, where he is currently pursuing a Ph.D. Since 1986, he has worked at Symbol Technologies in Holtsville, NY, and holds numerous patents in the bar code field.

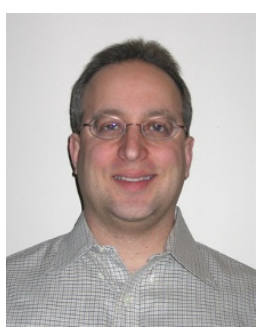

Joseph Katz is a Vice President of Mitsubishi Electric Research Laboratories and Deputy Director of its Research Laboratory. In addition to his role as a member of MERL's management team, he pursues his research interests in optoelectronics, communications, and RFID. Until 2004 he was with Symbol Technologies, where as a Senior VP of R\&D, he participated, initiated, and led projects in a wide range of technolo-

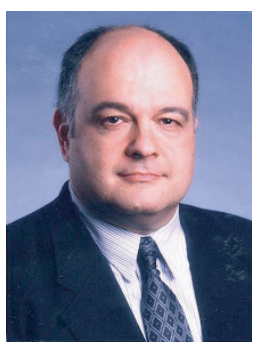
gies, including barcode/RFID data capture, optics, imaging, signal processing, computing, networking, security, biometrics, and communications. Prior to joining Symbol Inc., Joseph Katz worked at JPL/Caltech, where he conducted and led R\&D efforts in optical communications and advanced optoelectronic materials and devices, and was a recipient of the JPL Director's Research Achievement Award and of 25 NASA awards for technical innovations. Joseph Katz authored/coauthored over 150 technical publications and holds over 120 US patents. He was elected as a Fellow of both the IEEE and the Optical Society of America.

Leonard Bergstein, a holder of a Ph.D. degree in optics/electro-optics in 1960 from Polytechnic University, Brooklyn, NY, is a Professor Emeritus of electro-optical sciences at Polytechnic University. He is a Fellow of the Optical Society of America and has been a Consultant on optics and electronics to various industrial and educational organizations. He is one of the inventors of and holds basic patents on zoom

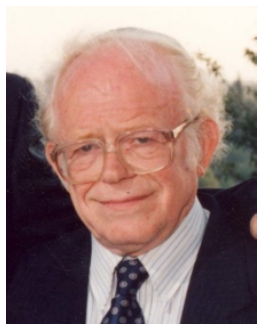
lenses and has written extensively on quantum electronics and electro-optics. 